\title{
Electronic coherences steer the strong isotope effect in the ultrafast Jahn-Teller structural rearrangement of methane cation upon tunnel ionization
}

\author{
Martin Blavier, ${ }^{1}$ Ksenia Komarova, ${ }^{2}$ Cayo E. M. Gonçalves, ${ }^{1}$ R. D. Levine, ${ }^{2,3}$ F. Remacle ${ }^{1,2}$ \\ ${ }^{1}$ Theoretical Physical Chemistry, University of Liège, 4000 Liège, Belgium \\ ${ }^{2}$ The Fritz Haber Research Center for Molecular Dynamics, The Hebrew University of \\ Jerusalem, 91904 Jerusalem, Israel \\ ${ }^{3}$ Department of Chemistry and Biochemistry and Department of Molecular and Medical \\ Pharmacology, David Geffen School of Medicine, University of California, Los Angeles, CA \\ 90095, USA
}

\begin{abstract}
We report on fully quantum electronic-nuclear dynamics following sudden ionization from the neutral in the three lowest electronic states of the $\mathrm{CH}_{4}{ }^{+}$and $\mathrm{CD}_{4}{ }^{+}$cations. There is a strong Jahn-Teller effect in the Franck-Condon region and we employ two nuclear degrees of freedom that span the internal coordinates involved in the Jahn-Teller coupling. The initial state results from tunneling ionization by a strong IR field which coherently pumps the three lowest states of the cation, $\mathrm{D}_{0}, \mathrm{D}_{1}$ and $\mathrm{D}_{2}$. The quantum dynamical simulations show that a strong isotope effect occurs when the ionization accesses significantly the $\mathrm{D}_{2}$ state of the cation in the FranckCondon region. The computed isotope effect is larger than expected on the basis of the effective mass ratio. The strong effect is due to fast oscillations of the electronic coherences between the $\mathrm{D}_{2}$ and the $\mathrm{D}_{1}$ and $\mathrm{D}_{0}$ electronic states and their modulation by the non adiabatic couplings before a significant onset of nuclear motion. The magnitude of the effect is similar to the one that we previously reported for a sudden photoionization process. A strong isotope effect has been observed in high harmonic spectroscopy studies of the very short time dynamics JahnTeller structural rearrangement of the methane cation upon sudden ionization.
\end{abstract}


TOC Graphics

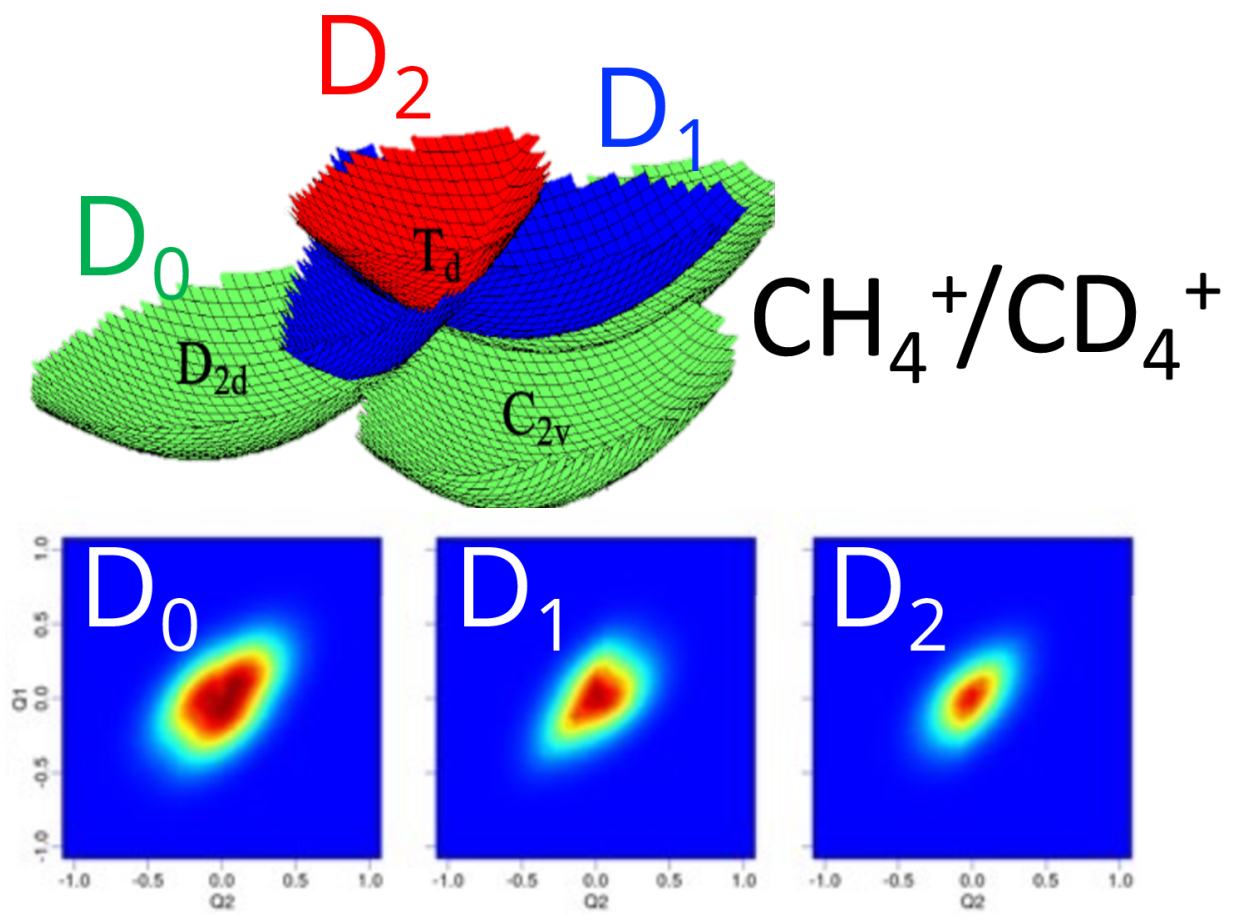




\section{Introduction}

The progress in the engineering of atto and few femtosecond optical pulses ${ }^{1-6}$ provides access to initiating, controlling and probing electronic coherences in molecular systems. ${ }^{7-10}$ Electronic coherences are built upon photoexcitation or photo or tunneling ionization when several electronic states are simultaneously and coherently accessed. This is made possible by the broad energy band width of short sub fs or atto pulses. The non stationary superposition of electronic and vibrational states built by the excitation process then evolves coherently on the potentials of the different electronic states where it encounters regions of non adiabatic couplings between electronic states that is driven by the nuclear motion. In this new intrinsically post Born and Oppenheimer regime, not only nuclei but also electrons are out of equilibrium in the initially pumped state. ${ }^{10-11}$ The new aspect brought by the post BornOppenheimer regime is the formation of electronic coherences at the excitation step which modulate the temporal and spatial localization of the electronic density in the molecule. As the dynamics unfold, new electronic states and new nuclear modes are brought in by the non adiabatic and anharmonic couplings. One of the challenges of attochemistry is to devise control schemes that exploit the electronic coherences built at the excitation step to steer the nuclei towards specific products.

A particularly interesting case for studying the role of electronic coherences built at the excitation step is when the regions of non adiabatic coupling are localized in the FranckCondon region or in its close vicinity. A text book example of such a situation is the Jahn-Teller rearrangement that occurs in the methane cation upon sudden ionization of the neutral. The sudden ionization creates a superposition of those three electronic states of the cation that are degenerate at the equilibrium $T_{d}$ geometry of the neutral molecule. In the cation, the $T_{d}$ geometry is unstable because of the Jahn-Teller (JT) effect which induces a structural rearrangement to a geometry of a lower symmetry. The rearrangement is ultrafast. It occurs in a very few fs's through strong non adiabatic couplings between the three electronic states in the Franck-Condon region where the electronic and nuclear motions are strongly correlated. The JT rearrangement is therefore significantly affected by isotopic substitution. A pioneering study by High Harmonic Spectroscopy indeed reports a strong isotope effect, larger than expected on the basis of the effective mass ratio, in the first $\approx 1.6 \mathrm{fs}$ of the dynamics. ${ }^{12-13}$ This experimental result stimulated several theoretical studies ${ }^{14-18}$ The yield in high harmonics has been shown to be proportional to the autocorrelation function, $|C(t)|^{2}$, of the initial state of the 
cation. ${ }^{19}$ The reason for the isotope effect is attributed to the slower motion out of the FranckCondon region of $\mathrm{CD}_{4}{ }^{+}$with respect to $\mathrm{CH}_{4}{ }^{+}$which leads to a higher yield in harmonics when the electron recombines with the cation. Theoretical studies therefore compare the ratio of the autocorrelation of the initial state of the two isotopomers to the ratio of the yields in harmonics. Mondal and Varandas reported MCTDH computations for a quasi vibronic coupling model in the three lowest states of the methane cation with increasing nuclear dimensionality up to the full 9 dimensionality. ${ }^{16-18}$ Their computations show that 5 nuclear degrees of freedom, the $t_{2}$ mode and the e mode of the $T_{d}$ geometry that are strongly involved in the Jahn-Teller effect are necessary to account for the large experimental isotope effect. ${ }^{18}$ In these computations, the ionization process is not described in details and the three electronic states are populated uniformly in the geometries of the Franck-Condon region for a fixed orientation of the molecule. Madsen ${ }^{15}$ studied the tunneling ionization rates and their angular dependence and confirmed the involvement of the same normal modes. Patchkovskii ${ }^{14}$ studied the role of the dependence of the early time dynamics on the gradient and the curvature of the potentials in the vicinity of the $T_{d}$ point on the large value of the ratio of the autocorrelation functions of $\mathrm{CH}_{4}^{+}$and $\mathrm{CD}_{4}^{+}$.

We recently reported on the isotope effect that occurs in the methane cation when the neutral is photoionized by a XUV attopulse. ${ }^{20}$ We carried out a fully quantum dynamical study in the three electronic states, using two nuclear coordinates described on a grid. The two coordinates span the normal modes of the molecule, with high weights on the two $t_{2}$ modes and the e mode involved in the JT distortion. The photoionization by the XUV attopulse was described by sudden ionization and the photoionization matrix elements are computed for each grid point geometry in the Franck-Condon region for an ensemble of 8000 orientations with respect to the electric field of the ionizing pulse. We considered two carrier frequencies of the pulse, which allows accessing the three lowest states of the cation in different amounts. A low carrier frequency $(\approx 13.65 \mathrm{eV})$ that corresponds to the $9^{\text {th }}$ harmonic of a $800 \mathrm{~nm}$ IR pulse leads to populations in the ground state $\mathrm{D}_{0}$ and the first excited state $\mathrm{D}_{1}$ of the cation, with a very low amount in $\mathrm{D}_{2}$. On the other hand, a carrier frequency that corresponds to the $11^{\text {th }}$ harmonics $(17.9 \mathrm{eV})$ accesses the three lowest states of the cation in roughly equal amounts. We showed by computing the ratio of the autocorrelation functions for an ensemble of randomly oriented molecules that a large isotope effect, with a ratio $\approx 2.6$ at $1.6 \mathrm{fs}$, is obtained for a photoionization by the higher frequency $h v_{11}$ attopulse, that accesses the $\mathrm{D}_{2}$ state. A lower isotope effect, with a ratio of 1.5 closer to the effective mass ratio $\left(\mu_{C-D} / \mu_{C-H}=1.86\right)$, is obtained for 
photoionization by the $h v_{9}$ attopulse, which does not significantly accesses the $\mathrm{D}_{2}$ state. We further showed that the autocorrelation function of the initial state being an observable sensitive on electronic coherences, that the larger isotope effect has a quantum nature and is due to the short time dynamics of the electronic coherences that involve the $\mathrm{D}_{2}$ state.

Here, we pursue our study of the role of electronic coherences in inducing large isotope effects, larger than the classical effect due to the different effective masses, by reporting fully quantal dynamical simulations following a sudden tunnelling ionizing effect, as is occurring in high harmonic spectroscopy. In order to assess the role of electronic coherences on the initial state that results from a tunneling ionization, we consider two field strengths of the IR pulse that generates the harmonics: a low strength of 0.03 a.u. $\left(3.1610^{13} \mathrm{~W} / \mathrm{cm}^{2}\right)$ that does not significantly access the $\mathrm{D}_{2}$ state in the Franck-Condon region and a larger field strength of 0.075 a.u. $\left(210^{14} \mathrm{~W} / \mathrm{cm}^{2}\right)$ that significantly accesses the $\mathrm{D}_{2}$ state. Our results show that as in the photoionization case, a large isotope effect of $\approx 2.7$ on the ratio of the autocorrelation functions is obtained for the stronger pulse, when the $\mathrm{D}_{2}$ state is accessed.

We have previously reported similar isotope effects that arise when an electronic coherence reaches a region of non adiabatic coupling in the diatomic molecules $\mathrm{N}_{2}$ and $\mathrm{LiH}^{21-23} \mathrm{We}$ showed using algebraic techniques for one nuclear dimension that the direction and the magnitude of the amplitude transfer when the electronic coherence reaches the coupling region is governed by the phase difference between the two electronic states present in the wave packet. The phase difference factorizes into a classical term that depends linearly on the reduced mass and a purely quantum part, that is non linear in the mass. ${ }^{24}$ The presence of this quantum term explains that the isotope effect is larger than the classical one when the vibronic wave packet is made of several electronic states.

We briefly report on the computational model in section 2, discuss the results for tunnel ionization in section 3 and provide a discussion of how the electronic coherences steer the isotope effect in section 4. Conclusions are given in Section 5.

\section{Computational details}

\subsection{Electronic structure and nuclear grid}

We use the same coordinates and the same level of electronic structure and the same potential energy surfaces (PES) as described in ref. ${ }^{20}$. The electronic structure is computed for the geometry of each grid point at the SA(3)-CASSCF $(9,8) / 6-31 \mathrm{G}++(2 \mathrm{df}, 2 \mathrm{pd})$ using MOLPRO, ${ }^{25}$ which provides the values of the potential energy for the three states, $D_{0}, D_{1}$ and $D_{2}$ as well as 
the non adiabatic couplings (NAC). The computed vertical IP at the $\mathrm{T}_{\mathrm{d}}$ geometry is $13.6 \mathrm{eV}$. The results of the electronic structure are in good agreement with ref. ${ }^{26}$. The larger atomic basis set allows for a good description of the ionization process.

The three lowest states of the methane cation, $\mathrm{D}_{0}, \mathrm{D}_{1}$ and $\mathrm{D}_{2}$, are unstable at the equilibrium geometry of the neutral ground state. The two nuclear coordinates of the grid, $\mathrm{q}_{1}$ and $\mathrm{q}_{2}$, are defined as linear combination of Cartesian displacements. They span the normal modes of the $\mathrm{T}_{\mathrm{d}}$ geometry that are involved in the JT effect, as well as the seams between the electronic states in the Franck-Condon region, where the geometry is of lower symmetry. The potential energy surface (PES) of the ground state, $\mathrm{D}_{0}$, exhibits two minima close to the FC region, the $\mathrm{C}_{2 \mathrm{v}}$ equilibrium geometry, and a secondary, less distorted, minimum of $\mathrm{D}_{2 \mathrm{~d}}$ geometry $\approx 0.28 \mathrm{eV}$ above the $C_{2 v}$ energy, see Figure 1. The coordinate $q_{1}$ points to the $C_{2 v}$ geometry and the coordinate $\mathrm{q}_{2}$ to the $\mathrm{D}_{2 \mathrm{~d}}$ one. To both, we added a small geometrical distortion in order to correctly describe the non adiabatic coupling (NAC) so that all geometries on the grid are of $\mathrm{C}_{1}$ geometry. We include 146 point along $\mathrm{q}_{1}$ and 184 along $\mathrm{q}_{2}$, which leads to 26864 grid points per electronic state. Figure 1 shows isocontours of the PES of $\mathrm{D}_{0}, \mathrm{D}_{1}$ and $\mathrm{D}_{2}$ for the $\mathrm{q}_{1}$ and $\mathrm{q}_{2}$ coordinates. In $\mathrm{CH}_{4}$, the $\mathrm{H}$ nuclei are equivalent. For a particular realization as shown in the insets of Figure 1, without the distortions added to $\mathrm{q}_{1}$ and $\mathrm{q}_{2}$ that takes the geometries of the grid points out of the $\mathrm{C}_{2 \mathrm{v}}$ geometry, we should have two equivalent $\mathrm{C}_{2 \mathrm{v}}$ minima and a $\mathrm{D}_{2 \mathrm{~d}}$. The distortion, that is needed to capture the NAC seams, lowers the symmetry of one of the $\mathrm{C}_{2 \mathrm{v}}$ minima. On the grid, the geometry closest to the global $\mathrm{C}_{2 \mathrm{v}}$ minimum $\left(\mathrm{q}_{1}=1.4, \mathrm{q}_{2}=0\right)$ has a root mean square deviation (RMSD) of 0.0418 Bohr. The $\mathrm{D}_{2 \mathrm{~d}}$ minimum $\left(\mathrm{q}_{1}=0, \mathrm{q}_{2}=-1.12\right.$, $\mathrm{RMSD}=0.0321 \mathrm{Bohr}$ ) is $0.165 \mathrm{eV}$ higher than that of the $\mathrm{C}_{2 \mathrm{v}}$ one and the more distorted $\mathrm{C}_{2 \mathrm{v}}$ $\operatorname{minimum}\left(\mathrm{q}_{1}=1.08, \mathrm{q}_{2}=1.26, \mathrm{RMSD}=0.1384\right.$, closer to a $\mathrm{C}_{\mathrm{s}}$ geometry $)$ is $0.1583 \mathrm{eV}$ higher than the $C_{2 v}$ minimum. $D_{1}$ exhibits three minima located close to the NAC seams while $D_{2}$ has a minimum at the $T_{d}$ point. The PES's of the three states have very different gradients around the $T_{d}$ point. The gradient of $D_{0}$ out of the unstable $T_{d}$ point is very steep but the minima around $D_{2 d}$ and $C_{2 v}$ are very shallow. $D_{1}$ is also unstable at the $T_{d}$ point, the $D_{1}$ PES exhibits three minima that are located close to the NAC seams with $\mathrm{D}_{0}$. The PES around the $\mathrm{T}_{\mathrm{d}}$ minimum on $\mathrm{D}_{2}$ is very steep. This implies that the wave packet transferred to $\mathrm{D}_{2}$ by the ionization process remains localized in this tight potential which makes the NAC with the $\mathrm{D}_{1}$ and $\mathrm{D}_{0}$ very efficient close to the $\mathrm{T}_{\mathrm{d}}$ geometry. On the other hand, the initial wave packets on $\mathrm{D}_{1}$ and $\mathrm{D}_{0}$ rapidly leave the region of the unstable $T_{d}$ geometry while the $D_{2}$ wave packet is trapped around the $T_{d}$ point. 

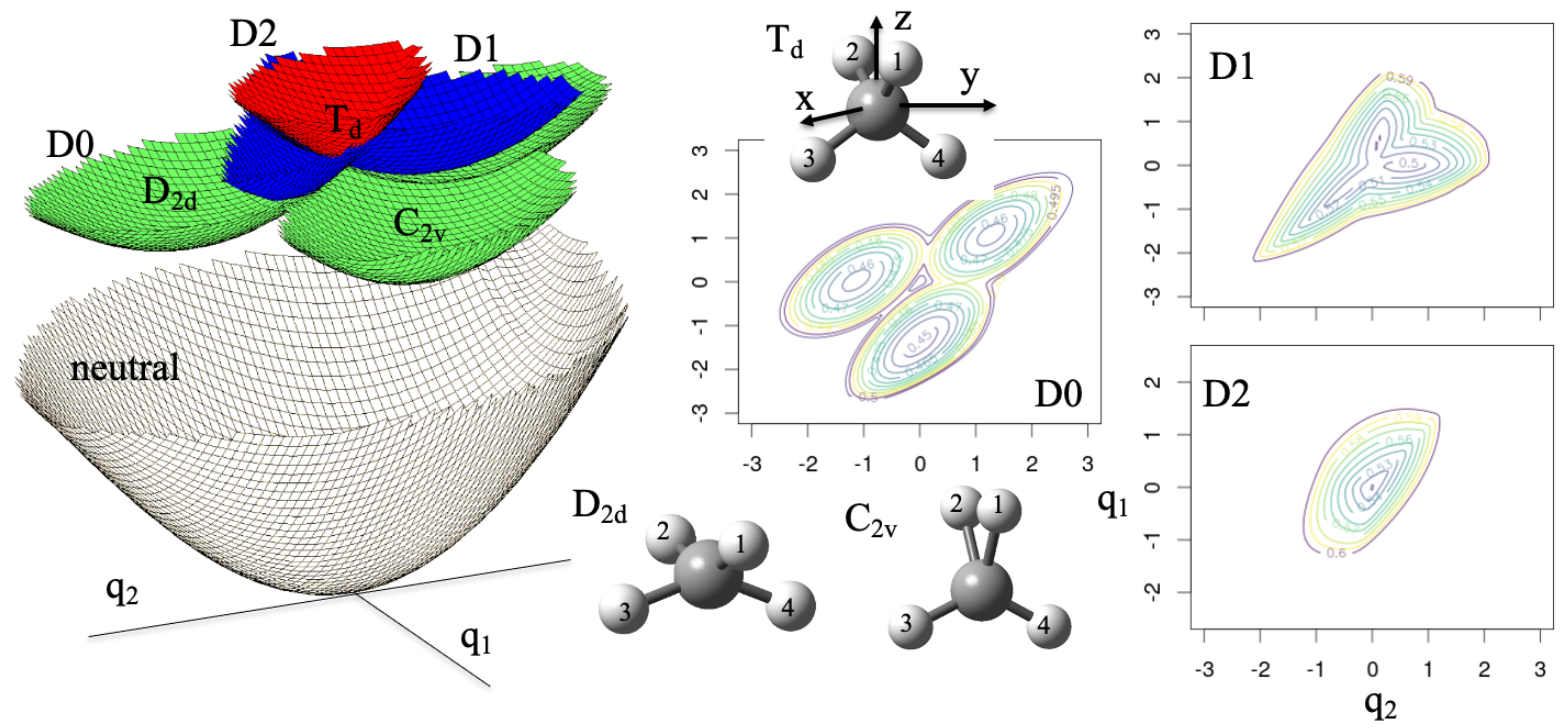

Figure 1: a) $3 \mathrm{D}$ view of the PES of the three lowest states of cation, $\mathrm{D}_{0}, \mathrm{D}_{1}$ and $\mathrm{D}_{2}$, computed at the SA-3-(9,8) /6-31G++(2df,2pd). B: Isocontours of the PES, $T_{d}$ geometry and geometries of the $\mathrm{D}_{2 \mathrm{~d}}$ and $\mathrm{C}_{2 \mathrm{v}}$ minima on $\mathrm{D}_{0}$. Isocontour spacing: $\mathrm{D}_{0} 0.01 \mathrm{eV} \mathrm{D} 1$. Isocontour spacing: $0.009 \mathrm{eV}$. C. D2. Isocontour spacing: $0.015 \mathrm{eV}$, and geometries on $\mathrm{D}_{0}$.

\subsection{Initial amplitudes in the states of the cation}

The tunneling amplitudes on the three states of the cation are determined within the three step model, ${ }^{27}$ assuming a tunneling ionization process at the maximum of the electric field for a random orientation of the molecular frame with respect to the laboratory frame. We use a MOADK model ${ }^{28-29}$ for describing the tunneling ionization from the neutral ground state to a particular electronic state of the cation, $D_{i}$, and a given orientation of the electric field, $\hat{\mathbf{e}}_{m}$, with respect to the molecular frame at the nuclear geometry of a given grid point $g$. We further assume that the structure parameters that appear in the expression of the molecular asymptotic wave function closely resembles the one electron density of the active electron that is ionized. ${ }^{30}$ The latter is given by the Dyson orbital, $\phi_{g, D_{i}}^{\text {Dyson }}(\mathbf{r})=\int d \mathbf{r}_{1} \ldots d \mathbf{r}_{N-1} \Psi_{g, D_{i}}^{c a t}\left(\mathbf{r}_{1} \ldots \mathbf{r}_{N-1}\right) \Psi_{g, G S}^{\text {neut }}\left(\mathbf{r}_{1}, \ldots, \mathbf{r}_{N}\right)$ with $\mathbf{r}$ the electronic coordinate and $N$ the number of electrons of the neutral. The strong field ionization probabilities, $W_{g, D_{i}}^{m}$ at each grid point to an electronic state of the cation $D_{i}$ for a given orientation of the electric 
field $\hat{\mathbf{e}}_{m}$ are then given as the product of the norm of the Dyson MO, $N_{g, D_{i}}=\int d \mathbf{r}\left|\phi_{D_{i}}^{D y s o n}(\mathbf{r})\right|^{2}$ multiplied by the Keldysh tunnel ionization rate,

$$
K_{g, D_{i}}^{m}=\exp \left(-\frac{2}{3} \frac{\left(2 I P_{g, D_{i}}^{m}\right)^{3 / 2}}{|\mathbf{F}|}\right)
$$

and weighted by the square modulus of the vibrational ground state wave function of the neutral at this grid point, $\left|c_{g, G S}^{\text {neut }}\right|^{2} 31$

$$
W_{g, D_{i}}^{m}=\left|c_{g, G S}^{\text {neut }}\right|^{2} N_{g, D_{i}} K_{g, D_{i}}^{m}
$$

In Eq.(1), $|\mathbf{F}|$ is the strength of the field. $I P_{g, D_{i}}^{m}$ is the IP at the geometry of grid point $g$ for a given orientation of the electric field, $\hat{\mathbf{e}}_{m}$, corrected by the Stark shift due to the strong electric field of the IR pulse on the neutral and on the cation.

$$
I P_{g, D_{i}}^{m}=E_{g, D_{i}}^{c a t}-E_{g, G S}^{n e u t}-|\mathbf{F}| \hat{\mathbf{e}}_{m} \cdot\left(\mu_{g, D_{i}}^{c a t}-\mu_{g, G S}^{n e u t}\right)
$$

where $\mu_{g, D_{i}}^{\text {cat }}$ and $\mu_{g, G S}^{\text {neut }}$ are the permanent dipoles of the cation state $D_{i}$ and of the neutral at grid point $g$. The Dyson MO's were computed as described in ref. ${ }^{32}$ and their norm computed from their expansion in AOs. At each grid point, the amplitudes of the initial state on each electronic state of the cation are proportional to the ionization rates

$$
c_{g, i}^{m} \propto \sqrt{W_{g, D_{i}}^{m}}
$$

We show the localization of the initial state as a heatmap in the $\left(\mathrm{q}_{1}, \mathrm{q}_{2}\right)$ coordinates in Figure 2 for $\mathrm{CH}_{4}{ }^{+}$for two values of the field strength: a low value of 0.03 a.u. $\left(3.1610-13 \mathrm{~W} / \mathrm{cm}^{2}\right)$, called weak field (wf) in the following and a higher value of 0.075 a.u. $\left(210^{14} \mathrm{~W} / \mathrm{cm}^{2}\right)$, called strong field (sf) in the following. This higher value corresponds to the peak power of the IR $800 \mathrm{~nm}$ pulse used to measure the high harmonic yields in ref. ${ }^{12}$. Each orientation $\hat{\mathbf{e}}_{m}$ defines a pure state. In a typical experiment, the orientation of the molecule is random with respect to the electric field. The results reported below are therefore computed for an ensemble of 8000 random orientations on unit sphere. 

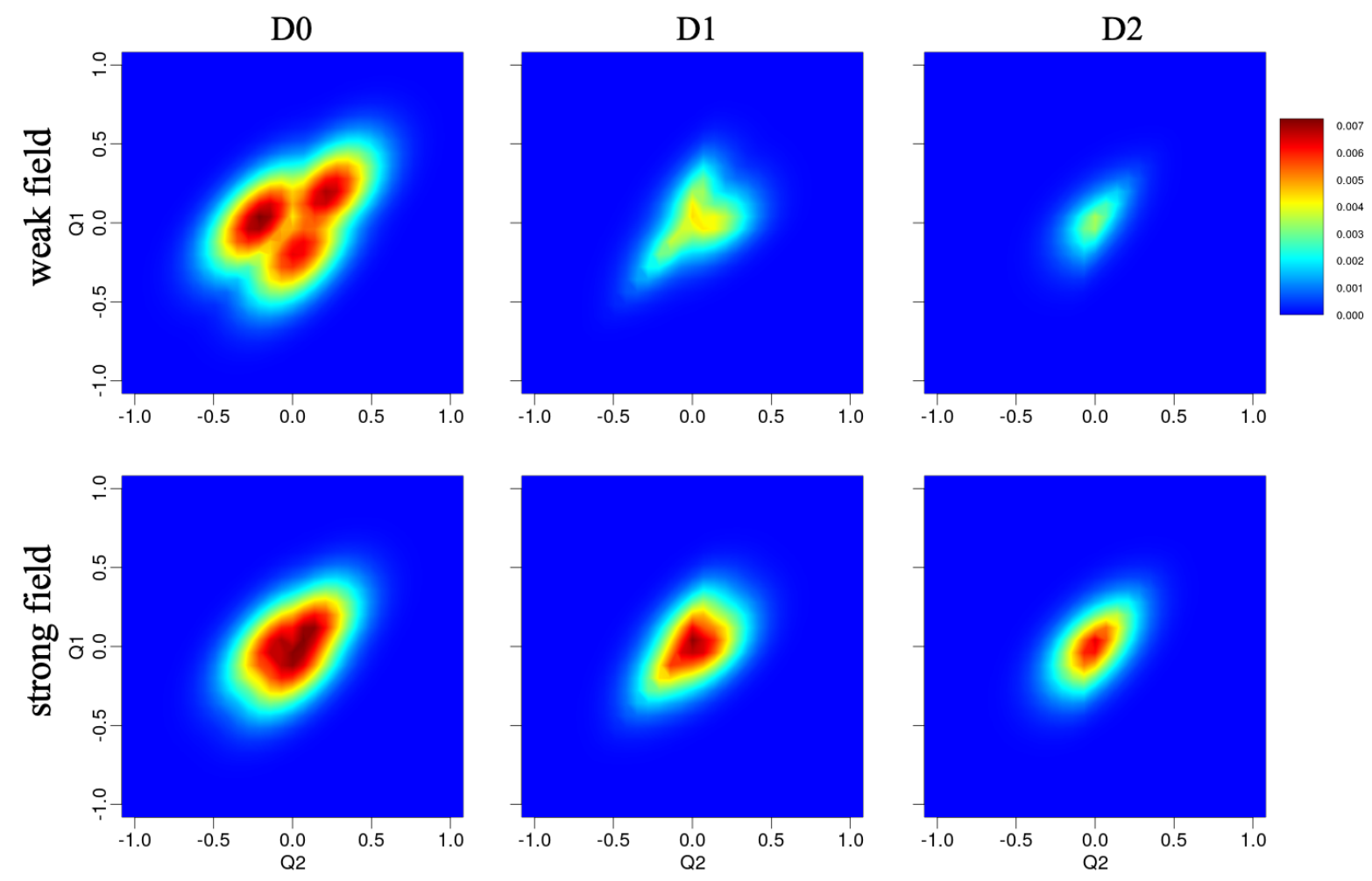

Figure 2. Localization of the initial state, $\left|c_{g i}\right|^{2}=\frac{1}{N} \sum_{m=1}^{8000}\left|c_{g i}^{m}\right|^{2}$ on the three electronic states, $\mathrm{D}_{0}, \mathrm{D}_{1}$ and $\mathrm{D}_{2}$ for $\mathrm{CH}_{4}{ }^{+}$computed for an ensemble of 8000 random orientations on the unit sphere for the weak field strength, top row and the strong field strength, bottom row. The localization of the $\mathrm{CD}_{4}{ }^{+}$initial states is shown in Figure $\mathrm{S} 1$.

The localization of the initial state on the three electronic states is shown in Figure 2 for the two field strengths for the methane cation $\mathrm{CH}_{4}{ }^{+}$. One can see that for the weak field, the localization of the initial state maps the isocontours of PES shown in Figure 1. The reason is that in the approximation we use, the dependence of the tunneling rate on the orientation comes from the Stark effect on the IP (Eq.(3)) which for this field strength is very weak. The tunneling rate then becomes determined by the field free energy difference between the neutral ground state and the electronic state of the cation at a given grid point. Since the PES of the neutral ground state is shallow in the Franck-Condon region, the variation of the IP on the grid is given by the energies of the cation states. The Stark effect is larger on the states of the cation than on the neutral GS. Therefore for higher field strengths, one obtains lower IP values than in the weak field case, which leads to a higher ionization yields, more initial populations in $\mathrm{D}_{1}$ and $\mathrm{D}_{2}$ and a more uniform localization of the initial states around the $T_{d}$ point. We obtain very similar initial localization of the initial states for $\mathrm{CD}_{4}{ }^{+}$see Figure $\mathrm{S} 1$ of the SI. The reason is 
that the only isotopic dependence of the initial state comes from the localization of the ground vibrational state of the neutral on the grid. The latter is slightly more localized around the $T_{d}$ geometry for $\mathrm{CD}_{4}{ }^{+}$than for $\mathrm{CH}_{4}{ }^{+}$.

\subsection{Vibronic Hamiltonian on the grid}

On the grid, the vibronic Hamiltonian used to propagate the principal component vectors, $\mathbf{U}_{\mathbf{s}}$, takes the form, see also Section S1 of the SI,

$$
\mathbf{H}_{i g, j g^{\prime}}=-\frac{1}{2} \mathbf{T}_{i g, j g^{\prime}} \delta_{i j}+\mathbf{V}_{i g, j g^{\prime}} \delta_{i j} \delta_{g g^{\prime}}+\left(\frac{1}{i} \tau_{i g, j g} \delta_{g g^{\prime}}\right) \cdot \mathbf{P}_{i g g g^{\prime}}
$$

The Hamiltonian includes the NAC coupling between each pair of electronic states. We neglect the second derivative NAC term which is much smaller. In the NAC term (last term of Eq. (5)), $i \neq j$.

In Eq. (5), the kinetic energy operator $\hat{T}$ in internal coordinates takes the form

$$
\hat{T}=-\frac{1}{2}\left(\frac{1}{\mu_{1}} \frac{\hat{\partial}^{2}}{\partial q_{1}^{2}}+\frac{1}{\mu_{2}} \frac{\hat{\partial}^{2}}{\partial q_{2}^{2}}+\frac{2}{\mu_{12}} \frac{\hat{\partial}^{2}}{\partial q_{1} \partial q_{2}}\right)
$$

and the components of the non-adiabatic coupling vector, $\tau_{i g, j g}$, are expressed in internal coordinates. The derivation and the expression of the reduced masses is given in the SI, as well as 2D maps showing the value of two components of the NAC coupling vectors, $\tau_{i g, j g}\left(q_{1}\right)$ and $\tau_{i g, j g}\left(q_{2}\right)$ on the grid for the three pairs of states. $\mathbf{P}$ is the momentum vector, whose two components are given by $\frac{\hbar}{i} \frac{\partial}{\partial q_{1}}$ and $\frac{\hbar}{i} \frac{\partial}{\partial q_{2}}$. We solve the time-dependent Schrödinger equation, $i \hbar \frac{d \mathbf{c}}{d t}=\mathbf{H c}$ numerically. The vector $\mathbf{c}$ is the vector of the complex amplitudes of the time-dependent wave function, $c_{i g}(t)$, on the grid. The non local kinetic energy and momentum matrix elements are computed using a finite difference algorithm, see SI. We report below the time-dependent value of the off diagonal term of the Hamiltonian averaged over grid points for each pair of electronic states: 


$$
H_{i j}(t)=\sum_{g} c_{i g}^{*}(t)\left(\frac{1}{i}\right) \tau_{i g, j g} \hat{P} \sum_{g^{\prime}} c_{j g^{\prime}}(t)
$$

2.4 Quantum dynamics on the grid averaged over random orientations

We report below results of the quantum dynamics averaged over 8000 random orientations of the electric field with respect to the molecule. We performed the averaging as in ref. ${ }^{20}$. The initial density operator, $\hat{\rho}^{\text {ens }}(0)$, of the resulting ensemble is given by summing over the pure states defined by the $M=8000$ orientations

$$
\hat{\rho}^{\text {ens }}(0)=\frac{1}{M} \sum_{m=1}^{M}\left|\Psi_{m}(0)\right\rangle\left\langle\Psi_{m}(0)\right|
$$

The initial density matrix on the basis of the $N_{\mathrm{e}}=3$ electronic states and $N_{\mathrm{g}}$ grid point can therefore be written as a quadratic form

$$
\rho^{e n s}(0)=\mathbf{A A}^{\dagger}
$$

where $\mathbf{A}$ is a rectangular matrix of dimensions $\left(N_{\mathrm{e}} \mathrm{x} N_{\mathrm{g}}=N_{\mathrm{T}}\right)$ x $M$ made of $M$ column vectors for each sampled orientation, $\mathbf{c}_{m}$, of $N_{\mathrm{T}} c_{i g}^{m}$ complex amplitudes. Since we compute $N_{g}=26864$ grid points per electronic state, $N_{\mathrm{T}} \gg>>M$. We showed in ref. ${ }^{20}$ that one can find the principal components of the matrix rectangular $\mathbf{A}$ by singular value decomposition:

$$
\mathbf{A}=\mathbf{U} \Sigma \mathbf{V}^{\dagger}
$$

The matrix $\Sigma$ is the diagonal matrix of the $M$ non zero eigenvalues of $\mathbf{A}$. $\mathbf{U}$ is a $N_{\mathrm{T}} \mathrm{x} M$ matrix whose columns are the left eigenvectors of $\mathbf{A}$ that correspond to a non zero eigenvalues. The $M \mathrm{x} M$ matrix $\mathbf{V}$ is the matrix whose columns correspond to the $M$ right eigenvectors of $\mathbf{A}$. One also shows that the matrix $\rho^{\text {ens }}(0)$ is rank deficient and has at most $M$ non zero eigenvalues with eigenvectors given by $\mathbf{U}_{\mathbf{s}}$. We can therefore exactly rewrite $\rho^{\text {ens }}(0)$ is a sum of $M$ 'pure states' which are its principal components:

$$
\rho^{\text {ens }}(0)=\mathbf{U} \Sigma \mathbf{V}^{\dagger} \mathbf{V} \Sigma \mathbf{U}^{\dagger}=\mathbf{U} \Sigma^{2} \mathbf{U}^{\dagger}=\sum_{s=1}^{M} \omega_{s}^{2} \mathbf{U}_{s} \mathbf{U}_{s}^{\dagger}
$$


To compute the average dynamics, it is therefore strictly equivalent to solve the time-dependent Schrödinger equation for the $M \mathbf{U}_{\mathbf{s}}$ vectors or for the $M: \mathbf{c}_{\mathbf{m}}$ ones. However, if the $\mathbf{A}$ matrix has a number of eigenvalues $L$ that are much larger than the rest of them, we can approximately write its expansion (Eq. (11)) in terms of $L$ principal components $\mathbf{U}_{\mathbf{s}}, L<<M$ :

$$
\rho^{e n s}(0) \approx \sum_{s=1}^{L} \omega_{S}^{2} \mathbf{U}_{s} \mathbf{U}_{s}^{\dagger}, L<<M
$$

In that case, propagating the $L$ principal components leads to a considerable saving of computer time. In ref. ${ }^{20}$, we could show analytically that for initial states produced by sudden ionization, there were exactly $L=3$ principal components of $\mathbf{A}$. In the case of initial amplitudes resulting from a sudden tunneling ionization process, we do not have such an analytical result because analytical dependence of the Keldysh tunneling ionization rates on the orientation of the field, see Eq. (1). However, the dynamics of the ensemble is recovered by three principal components which capture $99.97 \%$ of the trace of the ensemble density matrix for either one of the two isotopomers and for either of the two field strengths. More details are given in the SI.

We propagate the time-dependent Schrödinger equation using a finite difference algorithm for the kinetic energy and momentum operators for the three initial $\mathbf{U}_{\mathbf{s}}$ vectors. The probabilities for each initial state at each grid point are given by

$$
P_{i g}(t)=\rho_{i g, i g}^{e n s}(t)=\sum_{s=1}^{L} w_{s}\left|u_{i g}^{s}(t)\right|^{2}
$$

where $u_{i g}^{s}(t)$ are the components of $\mathbf{U}_{\mathrm{s}}(t)$ on the basis set and $w_{s}=\omega_{s}^{2} / \operatorname{Tr}[\rho(0)]$. The coherences terms of the ensemble density matrix take the form

$$
\rho_{i g, j g^{\prime}}^{e n s}(t)=\sum_{s=1}^{L} w_{s} u_{i g}^{s}(t) u_{j g^{\prime}}^{s}(t)=\sum_{s=1}^{L} w_{s} \rho_{i g, j g^{\prime}}^{s}(t)
$$

where $\hat{\rho}^{s}(t)=\left|U_{s}\right\rangle\left\langle U_{s}\right|$ is the density matrix corresponding to its principal vector eigenvector $\mathbf{U}_{\mathbf{s}}$.

The localization of the electronic coherence between electronic state $i$ and $j$ is diagonal in the grid index : 


$$
\rho_{i g, j g}^{e n s}(t)=\sum_{s=1}^{L} w_{s} u_{i g}^{s}(t) u_{j g}^{s}(t)=\sum_{s=1}^{L} w_{s} \rho_{i g, j g}^{s}(t) \quad i \neq j
$$

We report in the figures below the electronic coherences summed over all grid points

$$
\rho_{i j}^{e n s}(t)=\sum_{g}^{N_{g}} \sum_{s=1}^{L} w_{s} u_{i g}^{s}(t) u_{j g}^{s}(t)=\sum_{g}^{N_{g}} \sum_{s=1}^{L} w_{s} \rho_{i g, j g}^{s}(t), i \neq j
$$

The square modulus of the autocorrelation of the initial state takes the form:

$$
\begin{aligned}
& |C(t)|^{2}=\operatorname{Tr}\left[\rho^{e n s}(0) \rho^{e n s}(t)\right]= \\
& \sum_{i g}\left\langle i g\left|\sum_{s} w_{s}\right| u_{s}(0)\right\rangle\left\langle u_{s}(0)\left|\sum_{j g^{\prime}}\right| j g^{\prime}\right\rangle\left\langle j g^{\prime}\left|\sum_{s^{\prime}} w_{s^{\prime}}\right| u_{s^{\prime}}(t)\right\rangle\left\langle u_{s}{ }^{\prime}(t) \mid i g\right\rangle \\
& =\sum_{i g} \sum_{j g^{\prime}} \sum_{s} w_{s} u_{i g}^{s}(0) u_{j g^{\prime}}^{s}(0) * \sum_{s^{\prime}} w_{s^{\prime}}(0) u_{j g^{\prime}}^{s^{\prime}}(t) u_{i g}^{s^{\prime}} *(t) \\
& =\sum_{i g} \sum_{j g^{\prime}}\left(\sum_{s} w_{s} \rho_{i g, j g^{\prime}}^{s}(0)\right)\left(\sum_{s^{\prime}} w_{s^{\prime}} \rho_{j g^{\prime}, i g}^{s^{\prime}}(t)\right)
\end{aligned}
$$

So using Eq. (14), we see that

$$
\begin{aligned}
& |C(t)|^{2}=\operatorname{Tr}\left[\rho^{\text {ens }}(0) \rho^{\text {ens }}(t)\right]=\sum_{i g} \sum_{j g^{\prime}} \rho_{i g, j g^{\prime}}^{\text {ens }}(0) \rho_{i g, j g^{\prime}}^{\text {ens }} *(t) \\
& =\sum_{i g} \rho_{i g, i g}^{\text {ens }}(0) \rho_{i g, i g}^{\text {ens }}{ }^{*}(t)+\sum_{i} \sum_{g^{\prime} \neq g} \rho_{i g, i g^{\prime}}^{\text {ens }}(0) \rho_{i g, i g^{\prime}}^{\text {ens }}(t)+\sum_{i \neq j} \sum_{g^{\prime}, g} \rho_{i g, j g^{\prime}}^{\text {ens }}(0) \rho_{i g, j g^{\prime}}^{\text {ens }} *^{*}(t)
\end{aligned}
$$

is a direct probe of the electronic coherences (last term in Eq. (18)). The second term corresponds to vibrational coherences on the same electronic state. Since the yield in harmonics is proportional to $|C(t)|^{2},{ }^{19}$ the harmonic yield is an experimental probe of electronic coherences.

3. Results and discussion

3.1 Population and electronic coherences dynamics on the nuclear time scale 
The time evolution of the probabilities of each electronic state, $P_{i}(t)=\sum_{g=1}^{N_{g}} P_{i g}(t)$, was computed using Eq. (13) for tunneling initial states resulting from tunnel ionization by the weak field (Figure 3a) and by the strong field pulse (Figure 3b) for the two isotopomers.
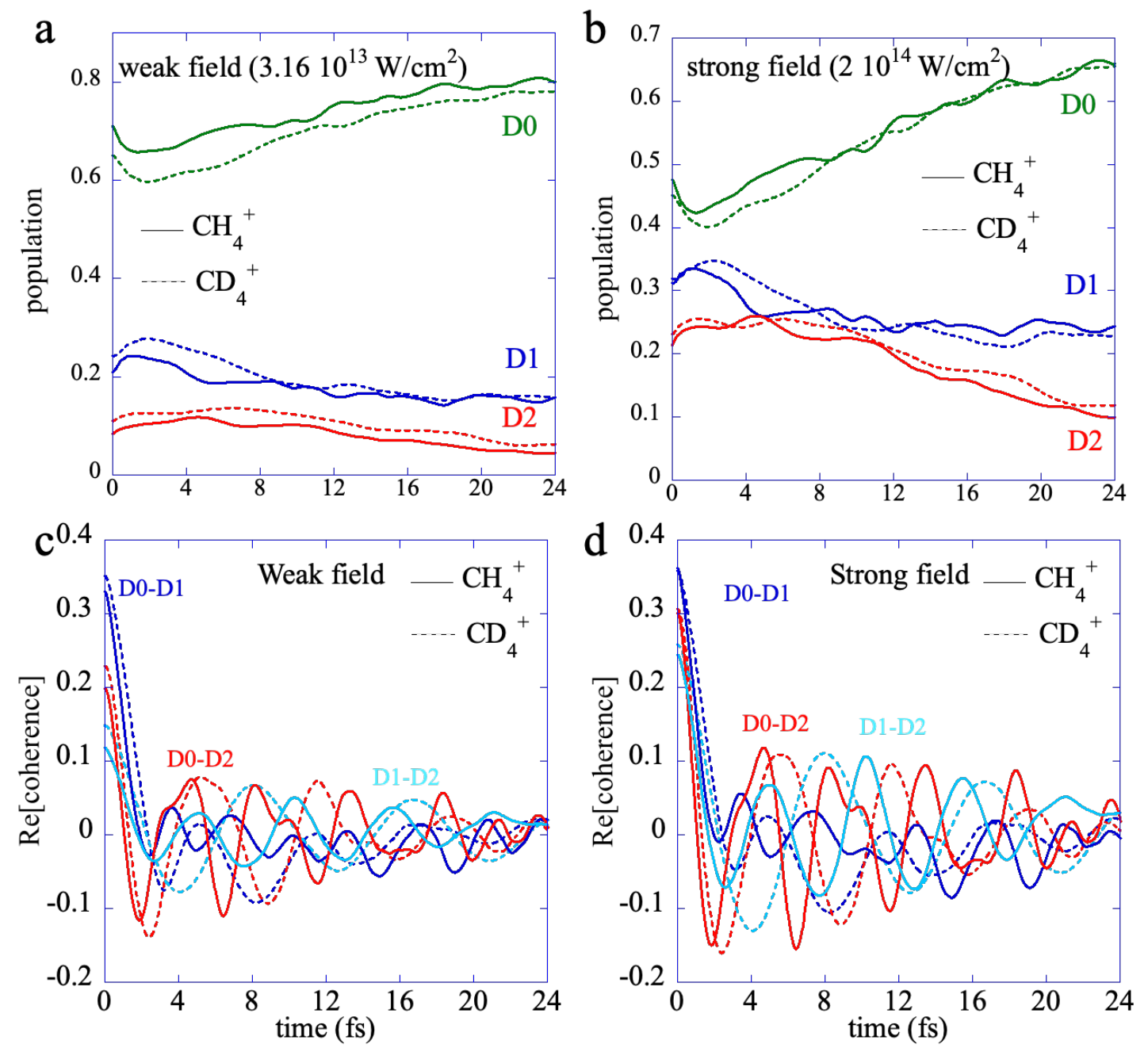

Figure 3. Panels a and b: Population dynamics, $P_{i}(t)$, for the ensemble of $\mathrm{CH}_{4}^{+}$(full lines) cations and $\mathrm{CD}_{4}^{+}$(dotted lines) upon tunneling ionization by a weak field (a) and a strong field (b). The population in $\mathrm{D}_{0}$ is plotted in green, in $\mathrm{D}_{1}$ in blue and in $\mathrm{D}_{2}$ in red. Panels $\mathrm{c}$ and $\mathrm{d}$ : Electronic coherence dynamics (Eq. (16)): c - weak field, $\mathrm{d}$ - strong field. The $\mathrm{D}_{0}-\mathrm{D}_{2}$ electronic coherence is plotted in red, the $\mathrm{D}_{0}-\mathrm{D}_{1}$ one in blue, and the $\mathrm{D}_{1}-\mathrm{D}_{2}$ coherence in azure. 
One can see from Figure 3 that the weak field strength $\left(0.03\right.$ a.u., $\left.3.1610^{13} \mathrm{~W} / \mathrm{cm}^{2}\right)$ pulse preferentially ionizes the ground state $\mathrm{D}_{0}$ (initial populations on $\mathrm{D}_{0}$ are 0.71 for $\mathrm{CH}_{4}{ }^{+}$and 0.65 for $\mathrm{CD}_{4}^{+}$). The initial population in $\mathrm{D}_{1}$ is 0.21 for $\mathrm{CH}_{4}{ }^{+}$and 0.24 for $\mathrm{CD}_{4}{ }^{+}$, about the double of that of $\mathrm{D}_{2}\left(0.08\right.$ for $\mathrm{CH}_{4}{ }^{+}$and 0.11 for $\left.\mathrm{CD}_{4}{ }^{+}\right)$. On the other hand, as discussed in section 2.3 above, the populations in $\mathrm{D}_{1}$ and $\mathrm{D}_{2}$ are larger for a tunnel ionization with the stronger pulse of 0.075 a.u. $\left(210^{14} \mathrm{~W} / \mathrm{cm}^{2}\right)$ : 0.31 for $\mathrm{CH}_{4}{ }^{+}$and 0.32 for $\mathrm{CD}_{4}{ }^{+}$in $\mathrm{D}_{1}, 0.21$ for $\mathrm{CH}_{4}{ }^{+}$and 0.23 for $\mathrm{CD}_{4}{ }^{+}$in $\mathrm{D}_{2}$ and 0.48 for $\mathrm{CH}_{4}{ }^{+}$and 0.45 for $\mathrm{CD}_{4}{ }^{+}$for $\mathrm{D}_{0}$. The tunneling ionization process creates a superposition of states for each orientation of the electric field which leads to electronic coherences at the level of the ensemble. The electronic coherences summed over all grid points, Eq. (16), are plotted in panels $\mathrm{c}$ and $\mathrm{d}$ of Figure 3. The oscillations in the populations are due to the non adiabatic transfers that are driven by the nuclear motion. One can see that the maximum of the oscillations of the population transfer coincide with large amplitudes of the oscillations of the electronic coherences. This is particularly clear at short time and for the oscillations of the $\mathrm{D}_{0}-\mathrm{D}_{2}$ electronic coherence. One also notices that oscillations of the electronic coherences in $\mathrm{CD}_{4}{ }^{+}$are slower than in $\mathrm{CH}_{4}{ }^{+}$and have a larger amplitude for the strong field initial states because there are more initial populations in the $\mathrm{D}_{2}$ and the $\mathrm{D}_{1}$ states. The oscillations of the electronic coherences are not periodic because of the strong NAC between the electronic states and the fact that the energy difference between the adiabatic electronic states varies rapidly in the FC region, see Figure 1. The coherences between the $\mathrm{D}_{2}$ and the two lower states $\mathrm{D}_{0}$ and $\mathrm{D}_{1}$ are sustained by the NAC as long as there is population in the $D_{2}$ state because the latter is trapped around the $T_{d}$ point on the steep $D_{2}$ potential. On the other hand, the wave packets on $D_{1}$ and $D_{0}$ are moving faster out of the $T_{d}$ region because of the steep gradient of the PES around $\mathrm{T}_{\mathrm{d}}$.

\subsection{Population and electronic coherences dynamics on the electronic time scale}

The short time behavior up to $2 \mathrm{fs}$ of the populations in the three electronic states and the electronic coherences (Eq. (16)) are shown in Figure 4 for the weak field (Fig. 4a) and the strong field (Fig.4b) initial states and the two isotopomers. Even on the short time (1.6 fs) probed by high harmonic spectroscopy using an $800 \mathrm{~nm}$ IR pulse, ${ }^{12-13}$ the populations in each electronic state start to vary since the initial state of the cation is localized around the $T_{d}$ point, where there is a strong non adiabatic coupling. The isotope effect on the populations is not very large. On the other hand, the periods of the oscillations of the electronic coherences are strongly affected by the isotopic substitution as shown in Figure $4 \mathrm{c}$ for the weak field initial state and 
in Figure $4 \mathrm{~d}$ for the strong one. On can see as expected that the dynamics of the $\mathrm{D}_{0}-\mathrm{D}_{1}$ coherence is not very much affected by the initial state while that of $D_{0}-D_{2}$ and $D_{1}-D_{2}$ coherence differ more because in the strong field case there is a larger amount of population initially present in $\mathrm{D}_{2}$.

We compare in Figures 4e and $4 \mathrm{f}$ the time evolution of the electronic coherences for weak and strong field for $\mathrm{CH}_{4}{ }^{+}$and $\mathrm{CD}_{4}{ }^{+}$respectively. The onset of the differences in the dynamics of the electronic coherences occurs earlier for $\mathrm{CH}_{4}{ }^{+}$than for $\mathrm{CD}_{4}{ }^{+}$. In this short time range, the nuclei have hardly time to move. We show in section 4 below that the difference is mainly due to the difference in the magnitude of amplitude transfers due to the non adiabatic couplings, in particular for the $\mathrm{D}_{1}-\mathrm{D}_{2}$ electronic coherence. The longer time dynamics is shown in Figure $\mathrm{S} 2$. One can see that the oscillations of the electronic coherences are getting slightly out of phase for the two field strengths as the dynamics unfolds, which is a manifestation of the effect of the amplitude transfers due to the NAC that involve the $\mathrm{D}_{2}$ state. This effect is larger for $\mathrm{CH}_{4}{ }^{+}$than for $\mathrm{CD}_{4}^{+}$. 

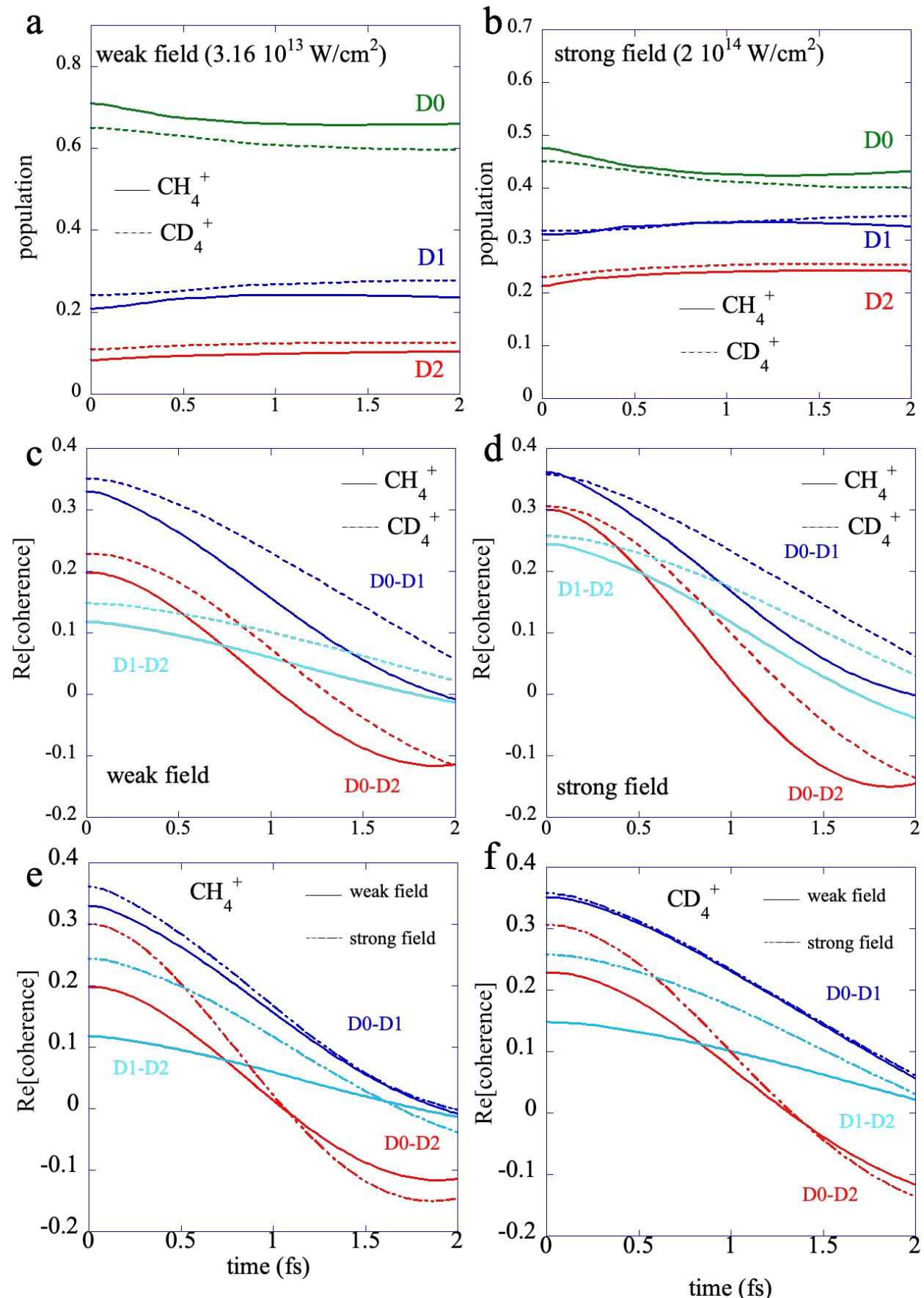

Figure 4. Panels a, b, c, d: same as Figure 3 for the short time range 0 to $2 \mathrm{fs}$. One can see that the $\mathrm{D}_{0}-\mathrm{D}_{1}$ coherence is not much affected by the localization of the initial state and the initial population in the $\mathrm{D}_{2}$ state while the $\mathrm{D}_{2}-\mathrm{D}_{0}$ and $\mathrm{D}_{1}-\mathrm{D}_{2}$ coherences are very much affected. Panels e and f: Comparison of the time evolution of the electronic coherences at weak and strong field for $\mathrm{CH}_{4}{ }^{+}$(e) and $\mathrm{CD}_{4}^{+}$(f). Here too, the biggest differences are for the $\mathrm{D}_{2}-\mathrm{D}_{0}$ and $\mathrm{D}_{1}-\mathrm{D}_{2}$ coherences. 
We next show in Figure 5a the auto correlation functions (Eq. (17)) and their ratio (Figure 5b) for the four cases. We obtain a ratio of the autocorrelation functions, $|C(t)|_{D}^{2} /|C(t)|_{H}^{2}$ at $1.6 \mathrm{fs}$ equal 2.4, in very good agreement with ref. ${ }^{12}$ for the high field strength initial state, and of 1.6 for lower field strength one. The large ratio of 2.4 computed for the high field strength initial state is due to the interplay between the electronic coherences that involve the $\mathrm{D}_{2}$ state and the onset of nonadiabatic interactions as the nuclei start to move. On the other hand, the ratio $|C(t)|_{D}^{2} /|C(t)|_{H}^{2}=1.6$ is essentially due to the isotope effect due to the $\mathrm{D}_{0}-\mathrm{D}_{1}$ coherence. This value is smaller but close to the ratio of 1.86 that one expects on the basis of the ratio of the effective masses of the $\mathrm{C}-\mathrm{D}$ and $\mathrm{C}-\mathrm{H}$ bonds. When the terms that involve the electronic coherences $D_{2}-D_{0}$ and $D_{2}-D_{1}$ in the last term of Eq. (18) are not included in the computation of the autocorrelation functions, dotted lines in Fig. 5b, the ratio $|C(t)|_{D}^{2} /|C(t)|_{H}^{2}$ is similar for the high and low field initial states (equal $\approx 1.6$ to strong field and 1.4 for weak field). The effect of the NAC is further seen by comparing Fig5a and 5c with Fig.5b and 5d which are computed without including the NAC term in the Hamiltonian, Eq. (5). In that case, the ratio for strong and weak field initial states is $\approx 1.3$ and it is 1.15 when the coherence terms due to the $\mathrm{D}_{2}$ are not included in the dynamics. 

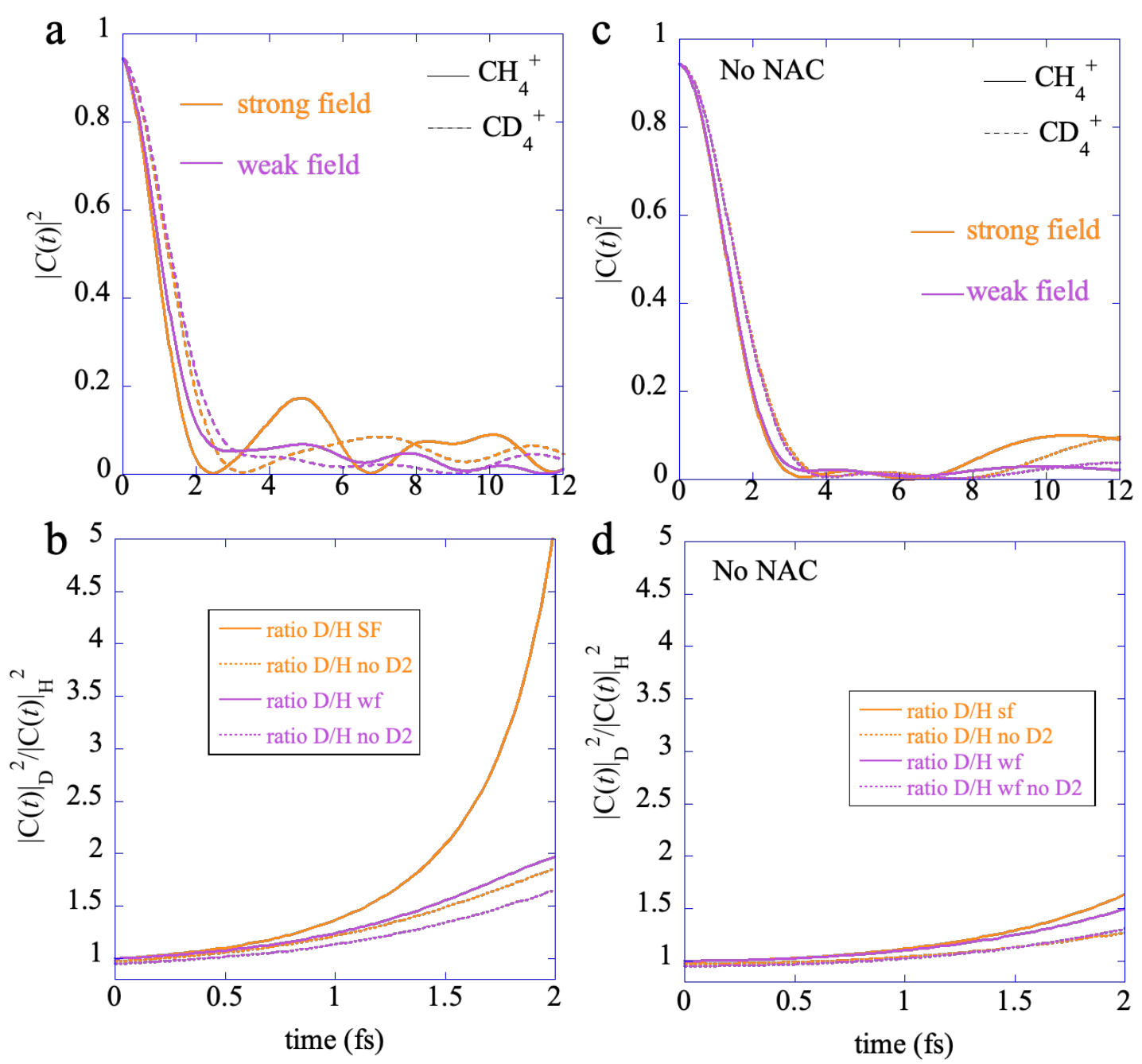

Figure 5.a) Autocorrelation functions, $|C(t)|^{2}$ (Eq. (17), of the weak field, (violet) and strong field (orange) initial states for $\mathrm{CH}_{4}^{+}$(full lines) and $\mathrm{CD}_{4}^{+}$(dashes) b) Ratio of the autocorrelation functions, $|C(t)|_{D}^{2} /|C(t)|_{H}^{2}$, computed exactly (full lines) and without included the coherent terms involving the $\mathrm{D}_{2}$ states (dots) c) Autocorrelation functions of the initial state without including the NAC in the Hamiltonian for computing the dynamics d) Ratio of the autocorrelation functions computed without NAC in the Hamiltonian.

\section{Discussion}


The results presented in section 3 point to a dominant role of the interplay between the electronic coherences and the non adiabatic coupling in getting an isotope effect different from that expected in the basis of the effective mass ratios. Indeed, the computed ratio of the masses in the kinetic energy term and in the NAC term in the Hamiltonian are $\approx 2$. The heat maps of the localization of the two components of the NAC vectors (Eq. S6), $\tau_{i j}\left(q_{1}\right)$ and $\tau_{i j}\left(q_{2}\right)$, at each grid point are plotted in Figures S3-S4 for $\mathrm{CH}_{4}+$ and Figure S5 for $\mathrm{CD}_{4}+$. In the FranckCondon region of the initial state population the seams of the NAC exhibit different localization for the $\mathrm{D}_{0}-\mathrm{D}_{1}$ and $\mathrm{D}_{1}-\mathrm{D}_{2}$ coupling (Figures $\mathrm{S} 4-\mathrm{S} 5$ ). The $\mathrm{D}_{0}-\mathrm{D}_{2} \mathrm{NAC}$ values on the grid are 50 times smaller than those of $D_{0}-D_{1}$ and $D_{1}-D_{2}$. The $D_{0}-D_{1}$ coupling is localized towards the positive values of the $\left(\mathrm{q}_{1}, \mathrm{q}_{2}\right)$ coordinates, while $\mathrm{D}_{1}-\mathrm{D}_{2}$ are primarily coupled towards the negative values of the $\left(\mathrm{q}_{1}, \mathrm{q}_{2}\right)$. This results in the population flow that is localized at specific zones in phase-space and it is responsible for the rapid reorganization of the population in all three electronic states (movies S1-S4 in the SI).

We show in Figure 6 heatmaps of the spatial localization of the electronic coherences in coordinate space and in Figure 7 in momentum, computed for the $\mathrm{D}_{0}-\mathrm{D}_{1}$ and $\mathrm{D}_{1}-\mathrm{D}_{2}$ electronic coherences, top row $\mathrm{CH}_{4}{ }^{+}$, middle row $\mathrm{CD}_{4}{ }^{+}$and bottom row $\mathrm{CH}_{4}{ }^{+}$without including the NAC term in the Hamiltonian. The patterns that develop as a function of time follow the patterns of the localization of the components of the NAC vector shown in Figure S6. They are slower to develop for $\mathrm{CD}_{4}{ }^{+}$than for $\mathrm{CH}_{4}{ }^{+}$as already seen in Figure 4. In the bottom row of Figure 6, computed for $\mathrm{CH}_{4}{ }^{+}$without including the NAC term, one sees that the localization of the electronic coherences hardly changes during the first 2 fs because the wave packets have hardly moved. This can be verified from the movies of the localization of the wave packets on $\mathrm{D}_{0}, \mathrm{D}_{1}$ and $\mathrm{D}_{2}$ computed with and without NAC for the two isotopomers (see SI). 


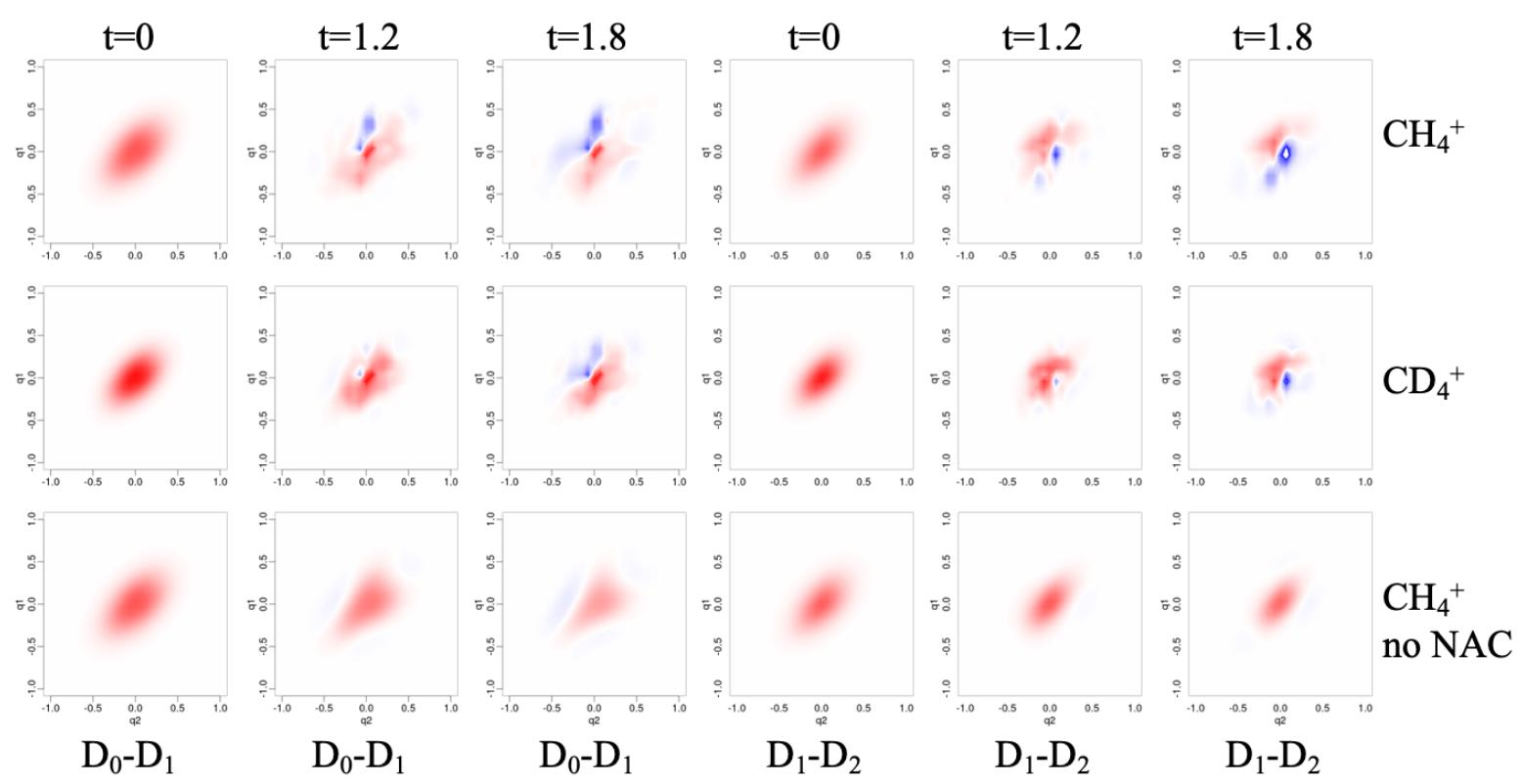

Figure 6: Heatmaps of the dynamics of the localization of the real part of electronic coherences, $\mathrm{D}_{0}-\mathrm{D}_{1}$ and $\mathrm{D}_{2}-\mathrm{D}_{1}$ at every grid point for the strong field case, computed for $\mathrm{CH}_{4}^{+}$(top row), $\mathrm{CHD}_{4}{ }^{+}$(middle row) and $\mathrm{CH}_{4}{ }^{+}$without including the NAC term in the Hamiltonian (bottom row). The time is in fs.

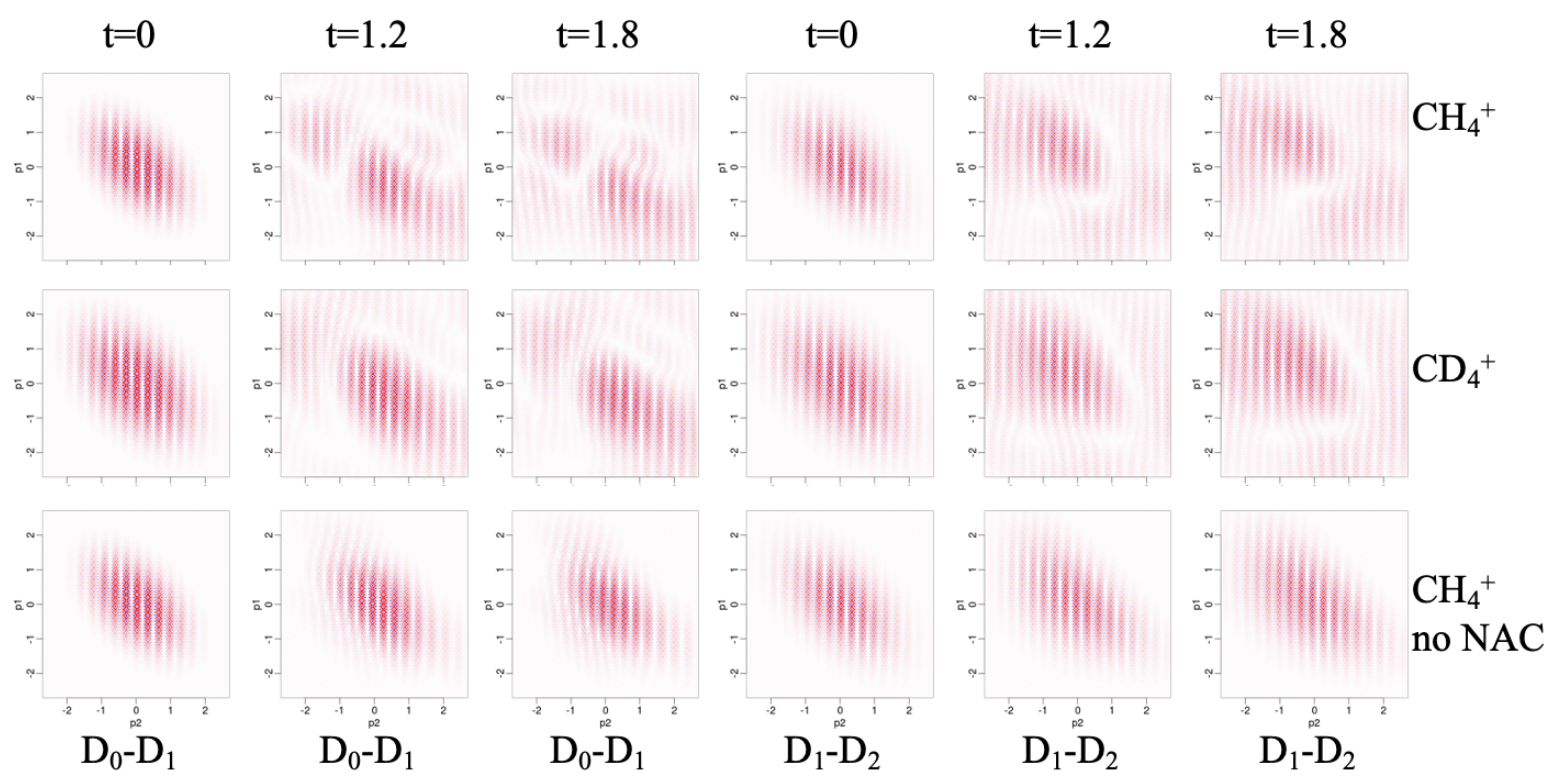

Figure 7: Heatmaps of the dynamics of the localization of the real part of electronic coherences, $\mathrm{D}_{0}-\mathrm{D}_{1}$ and $\mathrm{D}_{2}-\mathrm{D}_{1}$ in momentum space for the strong field case, computed for $\mathrm{CH}_{4}{ }^{+}$(top row), $\mathrm{CHD}_{4}{ }^{+}$(middle row) and $\mathrm{CH}_{4}{ }^{+}$without including the NAC term in the Hamiltonian(bottom row). 
We show in Figure 7 the localization of the electronic coherences in momentum space. The fast oscillations in momentum are due to the overall size of the coherence in space and they are slightly slower for $\mathrm{CD}_{4}{ }^{+}$than for $\mathrm{CH}_{4}{ }^{+}$. The development of smaller patterns in the momentum coherence maps results from the fragmentation of the electronic coherences due to the NAC term in the Hamiltonian.

The development of patterns of the electronic coherences in space and in momentum before the nuclei start to move is a manifestation of a purely electronic time scale due to the NAC coupling. They result from the interplay between the localization in space of the NAC seams shown in Figure S6 and the momentum term of the Hamiltonian, see Eq. (5). Since the NAC terms exhibit the expected ratio of $\approx 2$ at every grid point, the strong non classical isotope effect in the ratio of the autocorrelation functions plotted in Figure 5 arises from the momentum term. It is due to the fact that the tunneling ionization process creates a coherent superposition of the states of the cation. When the NAC term acts on an already existing coherence between the two states that are coupled, the direction and the magnitude of the amplitude transfer depend on the phase of the electronic coherence. We showed in refs. ${ }^{22-23}$ for diatomic molecules using a Condon approximation ${ }^{33}$ for the non adiabatic coupling that the transfer is maximum when the phase the momentum overlap between the wave packet on each coupled electronic state, $\langle i|\hat{P}| j\rangle$, is stationary. On the grid, we have an exact Condon-like separation at each grid point, see Eq. (5). The value of the NAC coupling, $H_{i j}(t)$, averaged over grid points between the wave packets on the two states that are coupled as a function of time is given by Eq. (7). Only the phases of the $\mathrm{D}_{1}-\mathrm{D}_{2}$ and $\mathrm{D}_{0}-\mathrm{D}_{1}$ coherences become stationary between 1 and $2 \mathrm{fs}$, and this only for the $\mathrm{q}_{2}$ components of the NAC vector $\tau_{i j}$. The values of the phase and the modulus of $H_{i j}(t)$ are plotted in Figure 8a for the weak field case and in Figure $8 \mathrm{~b}$ for the strong field case. The plots for the other coherences are given in Figure S6 of the SI. Movies of the rates of population transfer for the strong field initial state at each grid points are available in the SI. One can see from Figure 8 that the phases become stationary at earlier times for $\mathrm{CH}_{4}{ }^{+}$than for $\mathrm{CD}_{4}{ }^{+}$which explains the earlier onset of amplitude transfer for $\mathrm{CH}_{4}{ }^{+}$between these two pairs of states. One also see that the modulus of the coupling term is significantly larger for the $\mathrm{D}_{1}$ $\mathrm{D}_{2}$ coherence for strong field initial state, which explains the important role played by this coherence in the non classical strong isotope effect observed on the ratio of the autocorrelation 
functions in that case. Conversely, in the weak field case, one is getting an isotope effect smaller than expected on the mass ratio, which is essentially due to the $\mathrm{D}_{0}-\mathrm{D}_{1}$ electronic coherence. We note also that at short time, it is the component of the NAC vector along $\mathrm{q}_{2}$ that is pointing to the secondary $\mathrm{D}_{2 \mathrm{~d}}$ minimum that is dominant in the amplitude transfer.
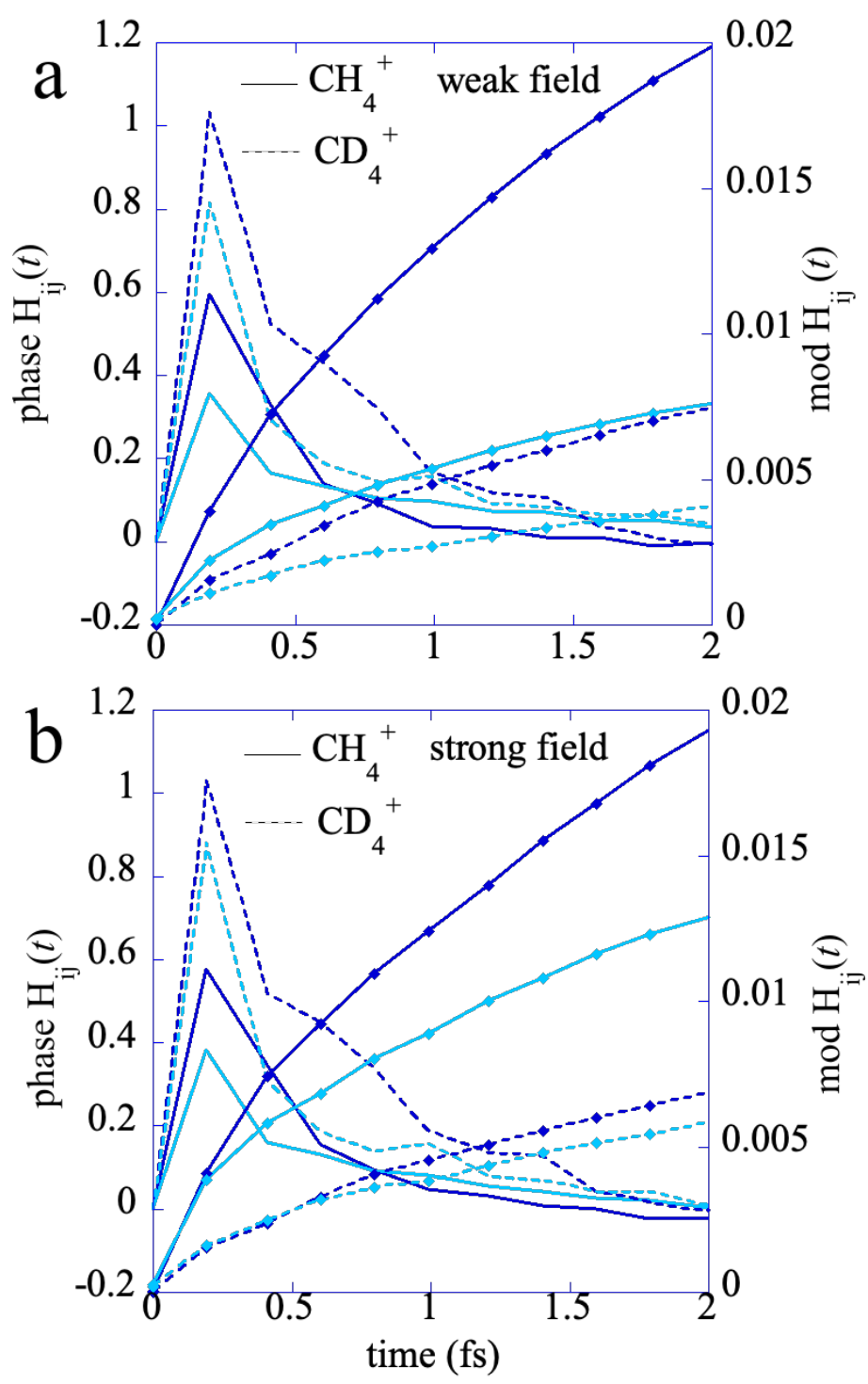

Figure 8. Early time evolution of the phases (left y axis) and moduli (right y axis) of NAC term $H_{i j}(t)$, Eq. (7), computed for the $\mathrm{q}_{2}$ component of the NAC vector for the weak field (panel a) and strong field (panel b) initial states for the two isotopomers $\left(\mathrm{CH}_{4}{ }^{+}\right.$in full lines and $\mathrm{CD}_{4}{ }^{+}$ 
in dashes). The moduli are plotted with diamonds symbols. The $\mathrm{D}_{0}-\mathrm{D}_{1}$ electronic coherence term is plotted in blue and the $\mathrm{D}_{1}-\mathrm{D}_{2}$ one in azure.

\section{Conclusions}

We investigated the very early time dynamics of the two isotopomers of the methane cation obtained by strong field ionization of the neutral ground state. We showed that the tunneling ionization prepares a superposition of the three lowest states of the cation. Two field strengths were considered, a weaker field that effectively does not access the higher $\mathrm{D}_{2}$ electronic state and a stronger one for which the $\mathrm{D}_{2}$ is significantly populated. The prompt electronic dynamics that follows the strong field ionization process exhibits a strong isotope dependence with a ratio of 2.4 in the autocorrelation functions, in agreement with the high harmonic spectroscopy experimental results which involve a tunneling ionization process. ${ }^{12-13}$ On the other hand, the dynamics for the weak field initial state leads to an isotope effect of 1.4 in the autocorrelation functions, which is smaller than the mass ratio. The magnitude of the effect is not changing upon averaging over the orientation of the electric field of the ionizing pulse with respect to the molecule. For both cases, we show that the isotope effect has a non classical origin and is due to the interplay between the dynamics of the electronic coherences and the amplitude transfers between each pair of electronic states due to the non adiabatic coupling. This non classical isotope effect occurs on a purely electronic time scale, before a significant onset of nuclear motion. We generalized the analysis of the isotope effect for systems in a coherent superposition of states developed for diatomic molecules ${ }^{21-24}$ to the present case of a $2 \mathrm{D}$ system, for which the NAC seams exhibit complex localization in space. We show by analyzing the phase and the modulus of the non adiabatic coupling between each pair of states that the $\mathrm{D}_{0}-\mathrm{D}_{1}$ and the $\mathrm{D}_{1}-\mathrm{D}_{2}$ electronic coherences play a major role in the non classical isotope effect. These are the coherences for which the phase of the coupling term, becomes stationary, which is the condition for an efficient amplitude transfer.

\section{Acknowledgements}

This work was supported by the Fonds National de la Recherche Scientifique (Belgium), F.R.S.-FNRS research grants \#T.0132.16 and \#J.0012.18. Computational resources have been provided by the Consortium des Equipements de Calcul Intensif (CECI), funded by the F.R.S.FNRS under Grant \# 2.5020.11. Support of the COST action Attochem(CA18222) is also 
acknowledged. We thank Dr. Stephan van den Wildenberg for the computation of the Dyson orbitals of methane.

References

1.Corkum, P. B.; Krausz, F., Attosecond Science. Nat. Phys. 2007, 3, 381-387.

2.Krausz, F.; Ivanov, M., Attosecond Physics. Rev. Mod. Phys. 2009, 81, 163-234.

3.Goulielmakis, E., et al., Single-Cycle Nonlinear Optics. Science 2008, 320, 1614-1617.

4.Calegari, F.; Sansone, G.; Stagira, S.; Vozzi, C.; Nisoli, M., Advances in Attosecond Science. J. Phys. B 2016, 49, 062001.

5.Galli, M., et al., Generation of Deep Ultraviolet Sub-2-Fs Pulses. Opt. Lett. 2019, 44, 13081311.

6.Hernández-García, C.; Popmintchev, T.; Murnane, M. M.; Kapteyn, H. C.; Plaja, L.; Becker, A.; Jaron-Becker, A., Isolated Broadband Attosecond Pulse Generation with near- and MidInfrared Driver Pulses Via Time-Gated Phase Matching. Opt. Express 2017, 25, 11855-11866. 7.Nisoli, M.; Decleva, P.; Calegari, F.; Palacios, A.; Martín, F., Attosecond Electron Dynamics in Molecules. Chem. Rev. 2017, 117, 10760-10825.

8.Lepine, F.; Ivanov, M. Y.; Vrakking, M. J. J., Attosecond Molecular Dynamics: Fact or Fiction? Nat Photon 2014, 8, 195-204.

9.Vrakking, M. J. J.; Lepine, F., Attosecond Molecular Dynamics. The Royal Society of Chemistry: Cambridge, 2019; p P001-500.

10.Remacle, F.; Levine, R. D., An Electronic Time Scale for Chemistry. Proc. Natl. Acad. Sci. USA 2006, 103, 6793-6798.

11.Muskatel, H. B.; Remacle, F.; Levine, R. D., The Post Born-Oppenheimer Regime: Dynamics of Electronic Motion in Molecules by Attosecond Few Cycle Spectroscopy. Phys. Scri. 2009, 80, 048101-7.

12.Baker, S.; Robinson, J. S.; Haworth, C. A.; Teng, H.; Smith, R. A.; Chirilă, C. C.; Lein, M.; Tisch, J. W. G.; Marangos, J. P., Probing Proton Dynamics in Molecules on an Attosecond Time Scale. Science 2006, 312, 424.

13.Marangos, J. P.; Baker, S.; Kajumba, N.; Robinson, J. S.; Tisch, J. W. G.; Torres, R., Dynamic Imaging of Molecules Using High Order Harmonic Generation. Phys. Chem. Chem. Phys. 2008, 10, 35-48.

14.Patchkovskii, S., Nuclear Dynamics in Polyatomic Molecules and High-Order Harmonic Generation. Phys. Rev. Lett. 2009, 102, 253602.

15.Madsen, C. B.; Abu-samha, M.; Madsen, L. B., High-Order Harmonic Generation from Polyatomic Molecules Including Nuclear Motion and a Nuclear Modes Analysis. Phys. Rev. A 2010, 81, 043413.

16.Mondal, T.; Varandas, A. J. C., The Jahn-Teller Effect in the Triply Degenerate Electronic State of Methane Radical Cation. J. Chem. Phys. 2011, 135, 174304.

17.Mondal, T.; Varandas, A. J. C., On Extracting Subfemtosecond Data from Femtosecond Quantum Dynamics Calculations: The Methane Cation. J. Chem. Theory Comput. 2014, 10, 3606-3616.

18.Mondal, T.; Varandas, A. J. C., Structural Evolution of the Methane Cation in Subfemtosecond Photodynamics. J. Chem. Phys. 2015, 143, 014304.

19.Lein, M., Attosecond Probing of Vibrational Dynamics with High-Harmonic Generation. Phys. Rev. Lett. 2005, 94, 053004.

20.Gonçalves, C. E. M.; Levine, R. D.; Remacle, F., Ultrafast Geometrical Reorganization of a Methane Cation Upon Sudden Ionization: An Isotope Effect on Electronic Non-Equilibrium Quantum Dynamics. Phys. Chem. Chem. Phys. 2021, 23, 12051-12059. 
21.Ajay, J. S.; Komarova, K. G.; Remacle, F.; Levine, R. D., Time-Dependent View of an Isotope Effect in Electron-Nuclear Nonequilibrium Dynamics with Applications to N2. Proc. Natl. Acad. Sci. U.S.A 2018, 115, 5890-5895.

22.Komarova, K.; vandenWildenberg, S.; Remacle, F.; Levine, R. D., Correlated ElectronNuclear Motion During Non-Adiabatic Transitions in Lih and Its Isotopomers. J. Phys. B 2020, $53,134001$.

23.Komarova, K. G.; Remacle, F.; Levine, R. D., Time Resolved Mechanism of the Isotope Selectivity in the Ultrafast Light Induced Dissociation in N2. J. Chem. Phys. 2019, 151, 114308.

24.Komarova, K.; Remacle, F.; Levine, R. D., The Density Matrix Via Few Dominant Observables: The Quantum Interference in the Isotope Effect for Atto-Pumped N2. J. Chem. Phys. 2021, 155, 024109.

25.Werner, H.-J.; Knowles, P. J.; Knizia, G.; Manby, F. R.; Schütz, M., Molpro: A GeneralPurpose Quantum Chemistry Program Package. Wiley Interdisciplinary Reviews: Computational Molecular Science 2012, 2, 242-253.

26.Frey, R. F.; Davidson, E. R., Potential Energy Surfaces of Ch+4. J. Chem. Phys. 1988, 88, $1775-1785$.

27.Corkum, P. B., Plasma Perspective on Strong Field Multiphoton Ionization. Phys. Rev. Lett. 1993, 71, 1994-1997.

28.Zhao, S.-F.; Xu, J.; Jin, C.; Le, A.-T.; Lin, C. D., Effect of Orbital Symmetry on the Orientation Dependence of Strong Field Tunnelling Ionization of Nonlinear Polyatomic Molecules. J. Phys. B 2011, 44, 035601.

29.Tong, X. M.; Zhao, Z. X.; Lin, C. D., Theory of Molecular Tunneling Ionization. Phys. Rev. A 2002, 66, 033402.

30.Ortiz, J. V., Dyson-Orbital Concepts for Description of Electrons in Molecules. J. Chem. Phys. 2020, 153, 070902.

31.Spanner, M.; Patchkovskii, S.; Zhou, C.; Matsika, S.; Kotur, M.; Weinacht, T. C., Dyson Norms in Xuv and Strong-Field Ionization of Polyatomics: Cytosine and Uracil. Phys. Rev. A 2012, 86, 053406.

32.van den Wildenberg, S.; Mignolet, B.; Levine, R. D.; Remacle, F., Temporal and Spatially Resolved Imaging of the Correlated Nuclear-Electronic Dynamics and of the Ionized Photoelectron in a Coherently Electronically Highly Excited Vibrating Lih Molecule. J. Chem. Phys. 2019, 151, 134310.

33.Condon, E. U., The Franck-Condon Principle and Related Topics. Am. J. Phys. 1947, 15, 365-374. 